\title{
Optimization studies for the coupling of bispecific antibodies to viral anchor molecules of a tumor vaccine
}

\author{
PHILIPPE FOURNIER ${ }^{1}$, MAXIMILIAN AIGNER ${ }^{1}$ and VOLKER SCHIRRMACHER ${ }^{1,2}$ \\ Tumorimmunology Program, German Cancer Research Center (DKFZ), \\ Im Neuenheimer Feld 280, 69120 Heidelberg, Germany
}

Received May 11,2010; Accepted June 14, 2010

DOI: 10.3892/ijo_00000772

\begin{abstract}
Tumor vaccines have to provide several signals for T cell activation. Among them, signal 1 (through TCR/ CD3) and signal 2 (through CD28) are the most important. We herein describe a procedure to introduce anti-CD3 and anti-CD28 signals into any tumor cell which is susceptible to infection by Newcastle disease virus (NDV). We developed the ATV-NDV tumor vaccine which consists of patientderived tumor cells (ATV) modified through infection by NDV. We tested for further improvement of vaccine efficiency the addition of two bispecific single-chain antibodies. They bind with one arm to the viral hemagglutininneuraminidase $(\mathrm{HN})$ or fusion $(\mathrm{F})$ protein of NDV expressed at the surface of the vaccine cells while the second arm is directed either against CD3 or CD28 of T cells. The aim of this study was to optimize the coupling of these new reagents to the tumor vaccine. When anti-CD3 and anti-CD28 molecules bind to the same anchoring viral molecule (e.g. $\mathrm{HN}$ ), competition for binding could occur under certain conditions. This was not the case when the bispecific reagents bound to separate viral molecules ( $\mathrm{HN}$ or $\mathrm{F}$, respectively). When using transfectants expressing $\mathrm{HN}$ and $\mathrm{F}$ either separately or on the same cell, we show that $\mathrm{T}$ cell activation works best when anti-CD3 and anti-CD28 are attached to the same stimulator cell. The clinical application of such a combined therapy with ATV-NDV vaccine cells and bi-specific antibodies allows to modify the strength of signal 1 and 2 in a quantitative and predictable way according to the immune status of the $\mathrm{T}$ cells and the requirements of the patients' immune system.
\end{abstract}

Correspondence to: Professor Volker Schirrmacher or Dr Philippe Fournier, German Cancer Research Center (DKFZ), Im Neuenheimer Feld 280, 69120 Heidelberg, Germany

E-mail: v.schirrmacher@dkfz.de

E-mail: p.fournier@dkfz.de

Present address: ${ }^{2} \mathrm{IOZK}$, Cologne, Germany

Key words: bispecific antibodies, antitumor, vaccination, signal 1, CD3, signal 2, costimulation, CD28, T cell activation and proliferation

\section{Introduction}

Patient-derived whole tumor cells are a good source of relevant tumor-associated antigens (TAAs) for a tumor vaccine, especially when they express unique tumor antigens that are characteristic of the individual tumor (1). However, the immunogenicity of such cells, i.e. their capacity to mount an immune response in the autochtonous host is usually low. Danger signals need to be introduced into tumor cells in order to achieve good vaccination effects. One strategy to induce or strengthen anti-tumor immune responses that we have investigated over the last 2 decades consists in the infection of the cells with an avian virus which improves the tumor cell immunostimulatory properties. This virus, the Newcastle disease virus (NDV), shows tumor selectivity in its replication behaviour and is therefore very well tolerated when applied to cancer patients.

In order to be able to exert effector functions, naive $\mathrm{T}$ cells require at least two signals: signal 1 through antigen recognition via the TCR and an additional co-stimulatory signal (signal 2). The most important $\mathrm{T}$ cell receptor for mediating a co-stimulatory signal is CD28. NDV infection of tumor cells results in upregulation of HLA-ABC (MHC class I) and in some cases of HLA-DR (MHC class II) molecules on tumor cells. In addition, the cell adhesion molecules ICAM-1 (CD54) and LFA-3 (CD58) are also upregulated (2). Thus, NDV-infected vaccine cells can present more efficiently MHC-bound peptides of TAAs and stimulate tumor-reactive T cells better than non-infected cells (3). ICAM-1 and LFA-3, which bind to LFA-1 and CD2 on the T-cell, are essential for the interaction of antigen-presenting cells with $\mathrm{T}$ cells $(4,5)$. They increase the contact between vaccine cells and $\mathrm{T}$ cells. Also, the neuraminidase (NA) activity of the HN molecule leads to a reduction of negative charge and thus diminishes the repulsion forces at the cell surface and causes a stabilization of the above-described intercellular interactions (6). In addition, the $\mathrm{HN}$ protein has been shown to act on the surface of cells as a B-7.1/B-7.2-independent co-stimulatory signal $(7,8)$. Since co-stimulatory signals from tumor cells are usually not provided by tumor cells, this is a key aspect in the effectiveness of the vaccine ATV-NDV.

Patients' $\mathrm{T}$ cells may vary in their immunocompetence. Some may have $\mathrm{T}$ cells which are anergic, especially with regard to their reactivity towards TAAs. Such anergic T cells may require a higher signal strength to become reactive 
again. The new second generation ATV-NDV vaccine we describe here allows to modify the strength of signal 1 and 2 through attachment of appropriate amounts of respective bispecific single-chain antibodies.

Besides the already described bispecific fusion proteins (bsHN-CD3 and bsHN-CD28) which are binding to the HN protein, we now describe new bsAbs (bsF-CD3 and bsF$\mathrm{CD} 28$ ) binding to the $\mathrm{F}$ protein of NDV. These fusion proteins were produced in a $\mathrm{CHO}$ mammalian expression system, purified and characterized especially for their binding to Ltk transfectants expressing $\mathrm{HN}$ or $\mathrm{F}$ proteins or both of them and to a human $\mathrm{T}$ cell line expressing CD3 and CD28 molecules.

We show in functional assays based on co-cultures of $\mathrm{T}$ cells with irradiated Ltk-transfectants expressing $\mathrm{F}$ proteins that crosslinking of these 2 cell populations, and thereby of CD3 molecules on $\mathrm{T}$ cells through addition of bsF-CD3 fusion proteins, induces $\mathrm{T}$ cell proliferation in a concentrationdependent manner. Under the conditions of a suboptimal signal 1, bsF-CD28 will be shown to be capable of providing co-stimulatory activity. Combinations of constructs binding to the 2 different viral molecules $\mathrm{HN}$ and $\mathrm{F}$, and thereby providing signal 1 and 2 avoided the risk of binding competition, led to strong $\mathrm{T}$ cell activation.

In addition, we demonstrate that signal 1 and 2 should be expressed at the surface of the same stimulator cell. The results demonstrate that signals mediated by anti-CD3 and anti-CD28 can be easily combined at the surface of the ATVNDV vaccine for optimal $\mathrm{T}$ cell activation and proliferation.

\section{Materials and methods}

Primary cells, cell lines and transfection. All cell lines were obtained from the tumor cell bank of the German Cancer Research Center (DKFZ, Heidelberg, Germany). Cell culture media were supplemented with $2 \mathrm{mM}$ L-glutamine, $10 \mathrm{mM}$ HEPES, $100 \mathrm{U} / \mathrm{ml}$ penicillin and $100 \mu \mathrm{g} / \mathrm{ml}$ streptomycin (all purchased from Gibco Invitrogen, Karlsruhe, Germany, unless otherwise mentioned). $\mathrm{CHO}$ dihydrofolate reductase (dhfr) deficient cells were grown in Iscove's modified Dulbecco's medium (IMDM) supplemented with 10\% FCS, $1 \times 10^{-4} \mathrm{M}$ hypoxanthine and $1.6 \times 10^{-5} \mathrm{M}$ thymidin. After transfection, these cells were cultured in $\alpha \mathrm{MEM}$ containing $5 \%$ dialyzed FCS (dFCS, Biochrom, Krefeld, Germany) and $10 \mathrm{nM}$ methotrexate (Calbiochem, Schwalbach, Germany). The human $\mathrm{T}$ cell lymphoma line Jurkat over-expressing CD28 (JCD28) and the adherent Ltk mouse fibroblasts were grown in RPMI-1640 medium with 5\% FCS (both from Gibco Invitrogen). Ltk-HN cells were derived from Ltk-Wt cells by transfection with HN cDNA of NDV (8) and after subcloning (Ltk-HN.A2, here called Ltk-HN). Ltk-F.B9 cells (F stably-transfected Ltk-Wt cells, here called Ltk-F) and Ltk-(HN+F).A5 cells [HN and F stably-transfected Ltk-Wt cells, here called Ltk-(HN+F)] were both elaborated during this study. Ltk cells were transfected by a method based on polyethyleneimine (jetPEI ${ }^{\mathrm{TM}}$, Polyplus, Strasbourg, France). Briefly, fresh, subconfluent cells were grown with FCScontaining medium. Two $\mathrm{ml}\left(4 \times 10^{5}\right.$ cells $)$ was placed in a $50 \mathrm{ml}$ centrifuge tube. In parallel, $6 \mu 1$ of the jetPEI solution was diluted in $100 \mu \mathrm{l}$ of sterile $150 \mathrm{mM} \mathrm{NaCl}$. This solution was added to $3 \mu \mathrm{g}$ plasmid DNA (diluted in a total of $100 \mu \mathrm{l}$ water) and mixed. Here, the DNA was complexed by the linear, cationic polyethyleneimine and thus its capacity by diffusion through biological membranes is greatly increased. The mixture was incubated for $30 \mathrm{~min}$ at room temperature before it was given slowly and dropwise to the cells. The cells were seeded immediately in a 6-well plate and incubated for $2 \mathrm{~h}$ at $37^{\circ} \mathrm{C}$. Finally, each well was replenished with $2 \mathrm{ml}$ of fresh medium. After transfection, the cells were cultured in selection medium, which (depending on cell type and transfected plasmids) was supplemented with $400 \mu \mathrm{g} / \mathrm{ml}$ geneticin (G418) or hygromycin B. Human PBMC were isolated either directly or from heparinized donor blood leucocytes (buffy coat), which were obtained from the blood bank, based in Heidelberg and Mannheim using a centrifugation step on Ficoll density gradient and washed at the end in RPMI without FCS. The isolation of T cells from PBMC was carried out by magnetic separation using the $\mathrm{T}$ Cell Negative Isolation Kit (Dynal, Hamburg, Germany). This isolation is based on superparamagnetic, monodisperse polystyrene particles with a uniform dispersion of magnetic material from $\gamma \mathrm{Fe}_{2} \mathrm{O}_{3}$ and $\mathrm{Fe}_{2} \mathrm{O}_{4}$. The particle surface is coated with a human IgG4 antibody, which specifically binds the Fc regions of mouse IgG antibodies. Next PBMC were adjusted to $1 \times 10^{8}$ cells $/ \mathrm{ml}$ in PBS. Then, $1 \times 10^{7}$ PBMC were mixed with $20 \mu 1 \mathrm{FCS}$ and $20 \mu 1$ antibody mix incubated for $20 \mathrm{~min}$ at $4^{\circ} \mathrm{C}$. The mix consisted of monoclonal antibodies for the surface proteins CD14, CD16a/b, CD56 and HLA II DR/DP, which bind to and mark all cells of the PBMC except the T-lymphocytes (B cells, NK cells, monocytes, activated T cells and granulocytes). After a washing step (with a centrifugation at $400 \mathrm{x} g$ during $8 \mathrm{~min}$ ), the cell suspension was added in $900 \mu \mathrm{l}$ $\mathrm{PBS} / 1 \times 10^{7} \mathrm{PBMC}$, and the magnetic particles were added as washed in PBS $\left(4 \times 10^{7}\right.$ particles $/ 1 \times 10^{7} \mathrm{PBMC}$ in $\left.100 \mu \mathrm{l}\right)$. This was followed by $30 \mathrm{~min}$ incubation with gentle shaking at room temperature. Subsequently, the resulting aggregates cell:particle were resuspended gently and the suspension was exposed to a magnetic field to separate the magnetically labeled cells from the $\mathrm{T}$ cells. The obtained cells were used for proliferation assays.

Virus and tumor vaccine. In this study the non-lytic, avirulent Ulster NDV strain, provided originally by Dr P. Russell (London, England) was used. It was propagated in eggs and purified on sucrose gradients as described (9). Quantification was performed by hemagglutination (HA) assay. The smallest virus concentration leading to visible agglutination of sheep erythrocytes is defined as 1 hemagglutination unit (HU). To prepare the ATV-NDV vaccine, the tumor cells are first inactivated by $\gamma$-irradiation. This led to the loss of the ability of the cells to divide, where the cells remained vital and could still be cultured for at least another three days without dying. The required amount of cells was irradiated in suspension (in 15 or $50 \mathrm{ml}$ centrifuge tube) with a ${ }^{137}$ Caesium source. For mouse cells, a dose of $100 \mathrm{~Gy}$ is used. The tumor cells are then modified through addition of NDV. To this end, tumor cells are washed with serum-free medium (250 x g, $5 \mathrm{~min})$, resuspended and counted. Cells at a concentration of $1 \times 10^{7}$ cells $/ \mathrm{ml}$ were then modified in serumfree medium with $100 \mathrm{HU}$ NDV. The incubation of tumor 
cell-virus suspension was carried out for one hour at $37^{\circ} \mathrm{C}$ and the mixture was shaken every $15 \mathrm{~min}$. The cells were then washed twice with medium $(250 \mathrm{x} \mathrm{g}, 5 \mathrm{~min})$ before use in the proliferation assays.

DNA expression vectors. Single-chain ( $\mathrm{scFv}$ ) antibodies specific for the $\mathrm{HN}$ and $\mathrm{F}$ proteins of NDV derived respectively from the HN hybridoma HN.B (Dr R.M. Iorio, Department of Molecular Genetics and Microbiology, University of Massachusetts Medical School, Worcester, MA, USA) and from spleen cells of a mouse that had been immunized against NDV, were cloned into the pERdhfr vector for expression in mammalian $\mathrm{CHO}$ cells using Integra flasks (Integra Biosciences, Fernwald, Germany). The scFv specific for CD3 were derived from the hybridoma TR66 (originally from Dr Antonio Lanzavecchia, Institute for Research in Biomedicine, Bellinzona, Switzerland). The CD28-specific $\mathrm{scFv}$ was derived from the 9.3 hybridoma kindly provided by Dr J.D. Hansen (US Geological Survey-Western Fisheries Research Center, Seattle, USA). Sequencings of the plasmids were carried out by the company GATC (Constance, Germany) and the raw sequences released online. The respective chromatograms were accordingly validated.

Antibodies. The following antibodies were used during this study: anti-HN (HN.B, mouse IgG2a, obtained from Dr R.M. Iorio anti-F (Icii, mouse IgG1, obtained from Dr R.M. Iorio), anti-His (mouse IgG1, from Dianova, Hamburg, Germany), anti-Histag (HIS1, mouse IgG2a, Sigma-Aldrich, Schnelldorf, Germany), anti-ETag (mouse IgG1, Amersham Biosciences, Freiburg, Germany), anti-FlagTag (M1, mouse IgG2b, SigmaAldrich), goat F (ab')2 anti-mouse biotin mouse IgG1 (only $\mathrm{H} \gamma$ 1) (Southern Biotech, Birmingham, USA), Streptavidin-PE (BD Pharmingen, Heidelberg, Germany), goat anti-mouse FITC mouse IgG2a (only $\mathrm{H} \gamma 2 \mathrm{a}$ ) (Southern Biotech), goat antimouse HRP (mouse IgG H+L), (Jackson ImmunoResearch, Cambridgeshire, England), goat $\mathrm{F}\left(\mathrm{ab}^{\prime}\right) 2$ anti-mouse IgM, IgG, IgA $(\mathrm{H}+\mathrm{L})$ labeled with PE (Southern Biotech).

Production and biochemical characterization of the purified $b s A b s$. The plasmids encoding the bispecific constructs bsHN-CD3 (no. 242) and bsHN-CD28 (no. 290) have been described previously (10). The plasmids encoding the proteins bsF-CD3 and bsF-CD28 were produced during this study. The amplification of the DNA plasmids was performed in TOP-10 bacteria (Gibco Invitrogen). Transfection of the plasmids into dCHO cells was carried out by electroporation (Biorad, Munich, Germany). Clones that were stably expressing the desired fusion protein were selected by limiting dilution. The production was conducted in high density cell culture systems (Integra Biosciences). The fusion proteins containing a $\mathrm{His}_{6}$-tag at their $\mathrm{C}$-terminus were purified by immobilized metal affinity chromatography; IMAC, as described (10). The E-tag containing protein was purified by means of an anti-E-tag immunoaffinity chromatography procedure (Amersham Biosciences).

Flow cytometry. Between $1 \times 10^{5}$ and $1 \times 10^{6}$ cells were given in a FACS tube and centrifuged $\left(250 \mathrm{x} \mathrm{g}, 3 \mathrm{~min}, 4^{\circ} \mathrm{C}\right)$ and incubated for $30 \mathrm{~min}$ on ice with the primary antibody in
$50 \mu 1$. The primary antibodies were diluted 1:100 in FACS buffer. The samples were then washed twice with $300 \mu 1$ FACS buffer $\left(250 \mathrm{x} \mathrm{g,} 3 \mathrm{~min}, 4^{\circ} \mathrm{C}\right)$ before they were resuspended in $50 \mu \mathrm{l}$ with the secondary fluorescently labeled antibody diluted 1:100 in FACS buffer. FACS buffer is PBS with $5 \%(\mathrm{v} / \mathrm{v})$ FCS solution and $0.1 \%(\mathrm{w} / \mathrm{v})$ sodium azide. The propidium iodide solution $(1 \mu \mathrm{g} / \mu \mathrm{l})$ was made in PBS at a final concentration of $1 \mathrm{ng} / \mu 1$.

Proliferation of immune $T$ cells. In order to analyze the proliferative potential of immune $\mathrm{T}$ cells, these cells were stimulated in a 96-well plate. For this purpose, $1 \times 10^{4}$ stimulator cells/well were added and incubated onto the plate in a volume of $50 \mu 1$ in the corresponding 10\% FCS-containing medium for at least $4 \mathrm{~h}$ to adhere. As stimulator cells were used the following irradiated transfectants [Ltk-HN, Ltk-F and Ltk-(HN+F)] cells or Ltk-Wt cells which were modified through infection with $100 \mathrm{HU}$ of NDV/107 cells. The fusion proteins were added at the required concentrations to the stimulator cells in the culture medium (containing 5\% FCS) in a volume of $50 \mu 1$. Finally, $1 \times 10^{5}$ immune cells were pipetted (in $50 \mu \mathrm{l}$ ) into the wells. The experiment was performed in triplicate and after incubation for $48 \mathrm{~h}$ at $37^{\circ} \mathrm{C}, 1 \mathrm{mCi}$ of ${ }^{3} \mathrm{H}$-thymidine was added into the wells. The cells were harvested after a further 18-h incubation and the incorporated radioactivity was measured in a liquid scintillation counter (Gammacell 1000 with a ${ }^{137}$ Caesium source, Gammamaster, Ede, The Netherlands).

\section{Results}

Elaboration and characterization of permanent Ltk cells expressing either $F$ or $H N$ and $F$ proteins as potential anchors for bispecific molecules. We prepared transfectants from the mouse fibroblast cell line Ltk. The starting material was the vector p36/7-HN which contains the HN cDNA (from NDV strain Beaudette C) between two Bam HI restriction sites (8). In parallel, we used the vector JMPpSFV1-F which contains the F-cDNA fragment (from NDV strain Ulster) between the $B s s \mathrm{HII}$ and $A p a \mathrm{I}$ restriction sites (11). The strategy we followed was to cut out the HN fragment from the p36/7-HN vector and replace it with the $\mathrm{F}$ fragment (originating from JMPpSFV1-F). To this end, two terminal linkers, each made of 2 hybridising oligonucleotides, were included to bridge the non-compatible interfaces. The orientation of the ligated fragment $\mathrm{F}$ was tested within plasmids of individual clones by digestion with HindIII (data not shown). To control the selected plasmids, these were digested with BamHI and the length of the insert was checked. At the end, sequencings of the terminal regions of the $\mathrm{F}$ inserts including the flanking linkers and vector sections were performed to ensure the precise ligation (data not shown). From one of the plasmids (p36/7-F, clone no. 22), a Maxiprep was prepared and the DNA introduced by electroporation into Ltk-Wt cells as described in Material and methods. Selection was carried out with Geneticin 418 (G418), which was added into the medium at a final concentration of $400 \mu \mathrm{g} / \mathrm{ml}$. After 5 sortings by flow cytometry for the expression of the F surface protein and an additional subcloning, a stable transfectant (Ltk-F clone B9) was obtained. Fig. 1A shows results from an 
A)

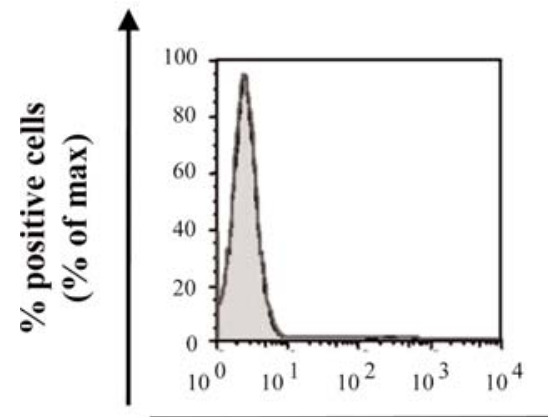

anti-HN
Ltk-F

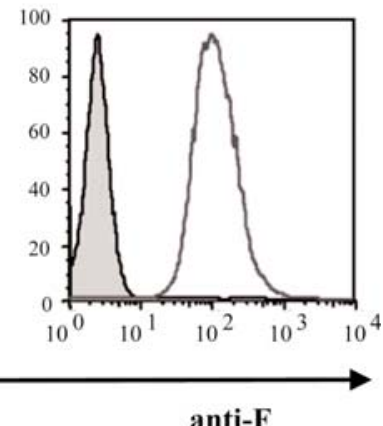

FL-2 (AFU)

B)

Ltk-HN-F

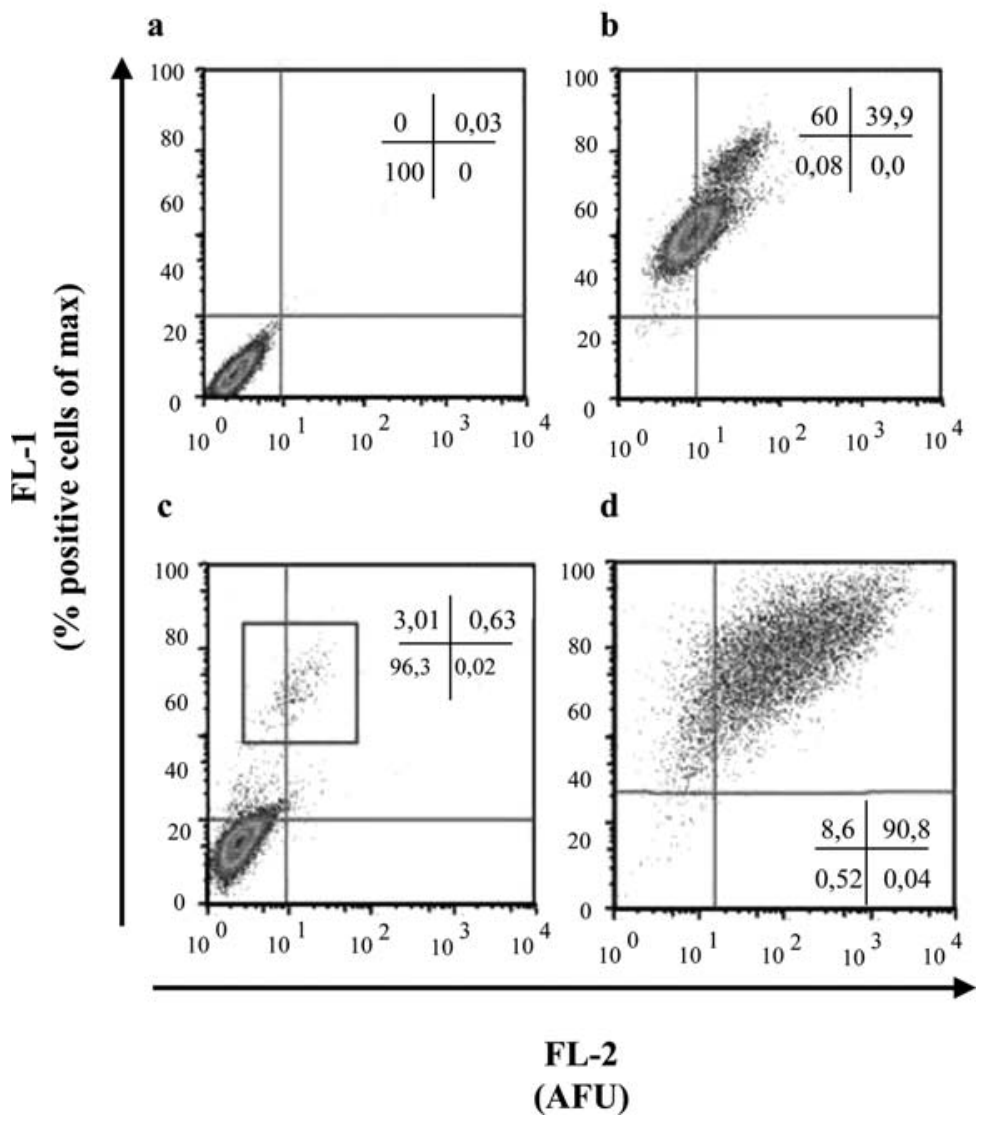

Figure 1. Cloning strategy for the construction of the plasmid p36/7-F and analysis of the Ltk-F and Ltk-(HN+F) transfectants. (A) High level expression of the F protein at the surface of the Ltk-F cells by flow cytometry. Ltk-F cells $\left(3 \times 10^{5}\right)$ which were obtained after transfection with the p36/7-F plasmid and after several cloning steps, were first labelled with the anti-HN mAb (HN.B; left) or with the anti-F mAb (Icii, right). The cells were then stained with PEconjugated goat anti-mouse secondary antibodies and analyzed by flow cytometry. Dead cells were excluded by propidium iodide staining. The negative control samples were obtained by incubation only with the secondary antibody (curve with gray shape). (B) High level expression of $\mathrm{HN}$ and F proteins at the surface of the Ltk $(\mathrm{HN}+\mathrm{F})$ cells. (a) Ltk-Wt cells $\left(1 \times 10^{5}\right)$, (b) Ltk-Wt cells infected with $400 \mathrm{HU}$ NDV Ulster/107 cells for $1 \mathrm{~h}$, (c) Ltk-Wt cells after transfection with the plasmids with pcDNA 3.1-neo (+)-HN and pcDNA 3.1-Hygro (+)-F coding, respectively, for the HN and F proteins, and (d) selected Ltk-(HN+F) double transfectants (clone A5). All cells were analysed by flow cytometry for surface expression of the HN and F proteins. (a and b) served as negative and positive controls for the staining. The square of the dot plot (c) highlights the Ltk cell population ( $1 \%$ of the total cells) which expressed $\mathrm{HN}$ and $\mathrm{F} 48 \mathrm{~h}$ after transfection. For staining, $3 \times 10^{5}$ cells were first incubated with anti-HN(HN.B) and anti-F(Icii) mAbs ( $1 \mu \mathrm{g}$ per sample). The binding of the anti-HN mAb was detected by a FITC-conjugated goat anti-mouse IgG2a isotype-specific secondary antibody whereas the anti-F mAb was detected at the cell surface with a biotinylated goat anti-mouse IgG1 isotype-specific secondary antibody and a PE-conjugated streptavidin as described in Material and methods. The expression of both signals on live cells was analyzed by flow cytometry. For all the stainings, dead cells were excluded using propidium iodide.

analysis by flow cytometry of this transfectant after staining with F- and HN-specific mAbs. This reveals that the cells express $\mathrm{F}$ but no $\mathrm{HN}$ at their surface.
Ltk cells co-expressing the $\mathrm{HN}$ and the $\mathrm{F}$ proteins of NDV were also established. For this purpose, we cloned the cDNA coding for $\mathrm{HN}$ and $\mathrm{F}$ in two different vectors harboring 

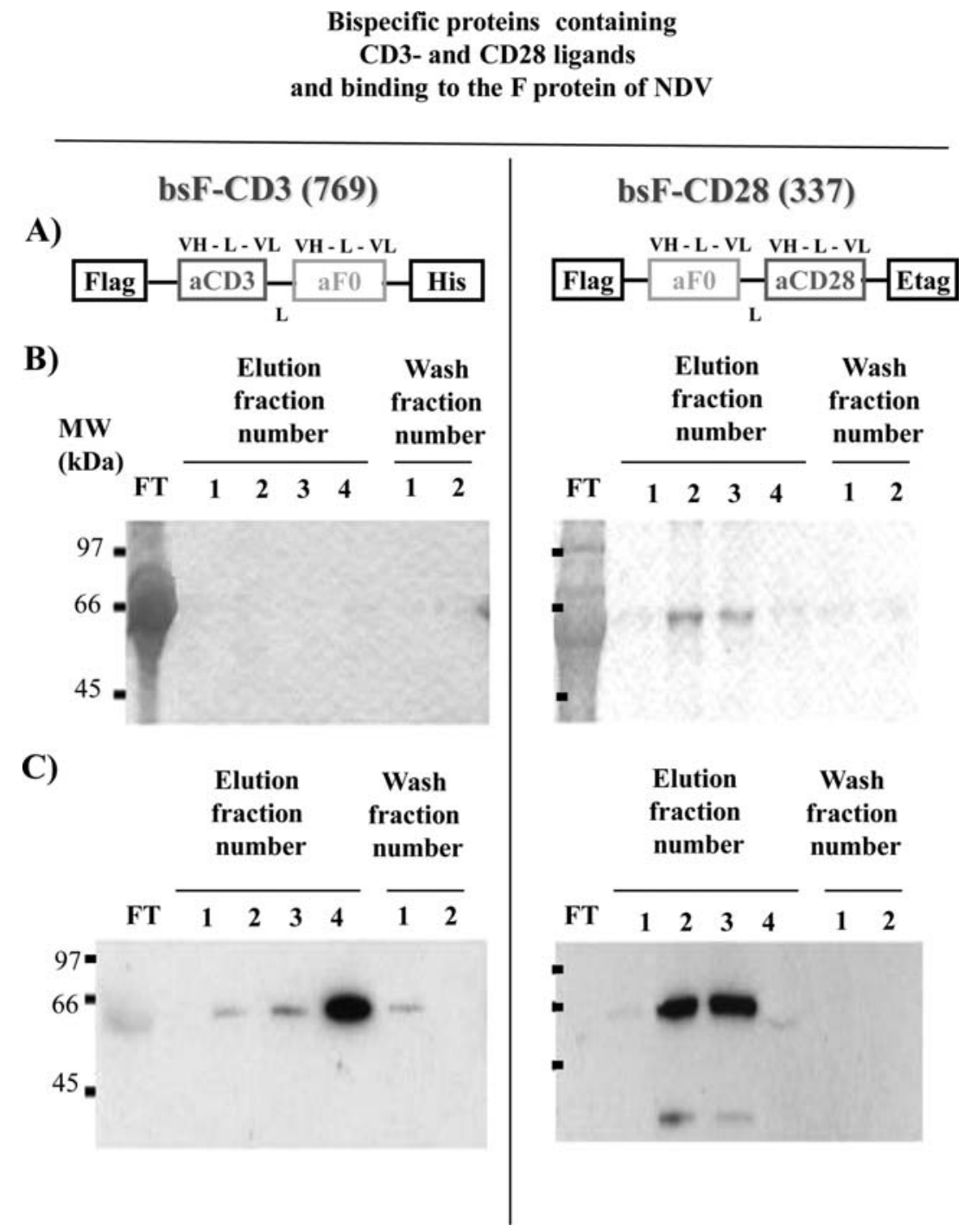

Figure 2. Biochemical characterization and analysis of the binding of the 2 constructs bsF-CD3 and bsF-CD28 binding, respectively, to CD3 or CD28 via one arm and to the viral F protein via the second arm. (A) Presentation of the 2 bsAb proteins bsF-CD3 and bsF-CD28 produced for this study. To construct the new plasmids (nos. 769 and 337), different scFvs were combined into the pERdhfr vector as described in Material and methods. The transcription of the bsAb proteins was carried out with the pERdhfr vector having the strong CMV (cytomegalovirus) promoter, RBS (ribosome-binding sites) sequences for the initiation of translation of proteins as well as the dhfr (dihydrofolate reductase) selection marker. (Left) The construct no. 769 contains a CD3-specific and an F-specific scFv antibody linked via a GlySer4 linker (L). Two flag sequences were added: one at the $\mathrm{C}$ terminus for the purification of the corresponding protein (His tag) and another one at the N terminus (Flag tag), allowing to detect the corresponding protein in Western Blot or by flow cytometry. (Right) The construct no. 337 contains a scFv specific for the F protein connected via L (a-15 amino acid long GlySer4 linker) to a scFv specific for $\mathrm{CD} 28$ derived from the 9.3 hybridoma. A Flag tag and an Etag sequence were inserted respectively at the $\mathrm{N}$ - and C-terminus of the recombinant bsAb protein for purification and detection in Western Blot and by flow cytometry. (B and C) Coomassie and Western Blot of the 2 purified bsAbs bsF-CD3 and bsF-CD28. The fusion protein no. 769 containing a C-terminal $\mathrm{His}_{6}$-tag was purified by Ni2 \pm NTA affinity chromatography whereas the fusion protein bsFo-CD28 by anti-E-tag immunoaffinity chromatography. After the purification of both proteins, eluted fractions were analyzed by SDS-PAGE, electroblotted onto nitrocellulose membranes and probed first with an anti-Flag-tag or anti-Etag mAb, and then with an HRP-conjugated goat anti-mouse IgG antibody. For that, $25 \mu 1$ of the individual fractions were mixed 1:2 with a reducing SDS sample buffer and incubated for $5 \mathrm{~min}$ at $95^{\circ} \mathrm{C}$. Then $20 \mu 1$ of each sample were applied on $12.5 \%$ polyacrylamide SDS-gels. The migration of the proteins could be visualized by Coomassie staining (B). The proteins of the second gel were transferred to a nitrocellulose membrane and detected with anti-Flag mAb. Using a peroxidase-coupled secondary antibody and the ECL substrate development, the proteins were made visible on X-ray film (C). FT, flow through.

2 different selection markers. Briefly, by means of digestion with BamHI from the vector p36/7-HN, HN was excised and ligated into the commercially available pcDNA 3.1-neo $(+)$ (with neomycin resistance). The orientation of the insert was checked with a XhoI digestion. The F fragment was obtained by double digestion of JMPpSFV1-F with BamHI and NotI. This DNA sequence was then unidirectionally inserted in the pcDNA 3.1-Hygro(+) vector (having a hygromycin resistance gene). The integrity of the insert was subsequently checked by double digestion with BamHI and NotI and by simple digestion with HindIII (data not shown). Both plasmids were then produced by Maxiprep and co-transfected into LtkWt cells using the jetPEI method. Forty-eight hours after transfection, a small percentage of the cells expressed both surface proteins as shown by flow cytometry (Fig. 1Bc). These were selected in medium supplemented with $400 \mu \mathrm{g} / \mathrm{ml} \mathrm{G} 418$ and hygromycin $\mathrm{B}$. The selection of a stable Ltk transfectant (clone A5) that expresses both surface antigens $\mathrm{HN}$ and $\mathrm{F}$ at 
D)

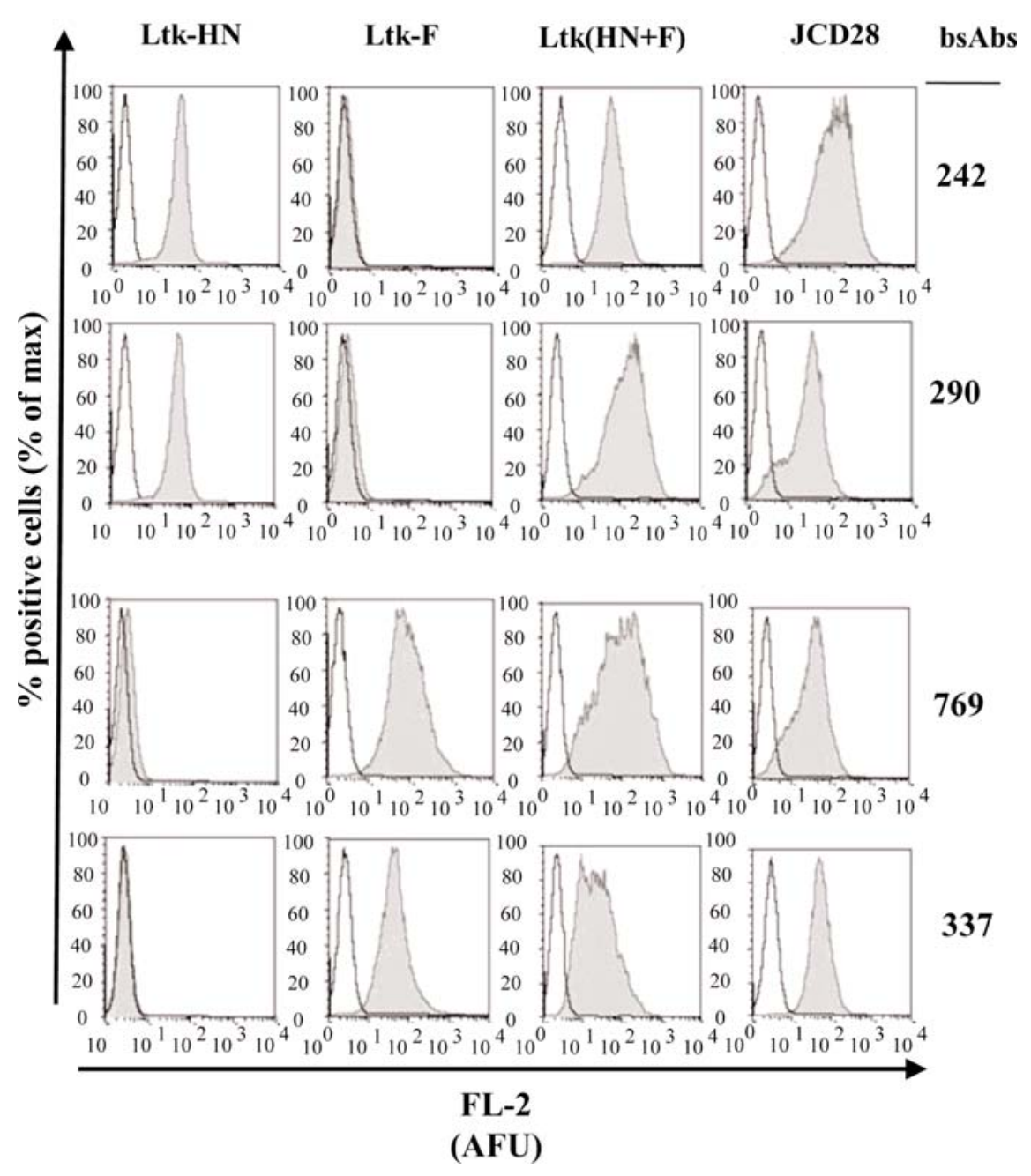

Figure 2. Continued. (D) Binding specificities of the two HN (top) and $2 \mathrm{~F}$ (bottom)-specific bsAb proteins. Cells of the different cell lines (1x105), Ltk-HN, Ltk-F, Ltk-(HN+F) or JurkatCD28, were incubated for 45 min with $1 \mu \mathrm{g}$ of the different tested proteins: top, bsHN-CD3 (no. 242 ) and bsHN-CD28 (no. 290 ); bottom, bsF-CD3 (no. 769) and bsF-CD28 (no. 337). The staining was carried out in a first step with anti-His or anti-E-tag mAbs and then in a second step with PE-conjugated goat anti-mouse secondary antibody. As a negative control samples were stained only with the secondary antibodies. Dead cells were excluded by staining with propidium iodide during all the FACS measurements.

high levels was obtained after 11 FACS sortings and a subsequent FACS subcloning. Fig. 1B shows double staining with $\mathrm{HN}$ - and F-specific mAbs of the cells without transfection (a), directly after transfection (c) and after selection and cloning (d). (b) shows for comparison Ltk-Wt cells infected for $1 \mathrm{~h}$ with $400 \mathrm{HU}$ NDV per $10^{7}$ cells.

Construction, production, purification and biochemical characterisation of the proteins bsF-CD3 and bsF-CD28. The plasmid constructs bsF-CD3 (no. 769) and bsF-CD28 (no. 337) were obtained by combining the different singlechain antibody fragments ( $\mathrm{scFv}$ ) coding for the different antigens in a CHO-based mammalian expression system. They were examined for their integrity by restriction digestion and sequencing. The selected plasmids bsF-CD3 and bsF-CD28 were then transfected by electroporation into $\mathrm{CHO}$ cells. Stable clones producing the desired proteins were then etablished by subclonings and cultured in high density cell culture for the production of the corresponding proteins. A volume of $15-30 \mathrm{ml}$ of culture supernatant containing the corresponding soluble fusion protein was obtained weekly per Integra flask. For purification of bsF-CD3 (and bsHNCD3), the culture supernatants were dialyzed and loaded onto a nickel-NTA-Sepharose column to which the bsAb fusion proteins bound via their $\mathrm{C}$-terminal $\mathrm{His}_{6}$-tag. After one or several washing steps, the proteins were eluted from the column by increasing concentrations of imidazol. These fractions were collected and analyzed by SDS-PAGE and subsequent Coomassie staining. After transfer onto a membrane, the specific protein could be visualised by antibody staining via Western blot. This allowed to evaluate the quantity and purity of the new fusion proteins. The proteins bsF-CD28 and bsHN-CD28 were purified by immunoaffinity using an anti-E-tag column. The fractions with the highest and purest protein were mixed, dialyzed against PBS, sterile filtered and stored at $-80^{\circ} \mathrm{C}$ until use.

The F-specific fusion proteins no. 769 and 337 appear to be produced at a much lower production yield than the 
Table I. Summary of the characteristics of the 4 purified fusion proteins used in this study.

\begin{tabular}{llll}
\hline Protein no. & \multicolumn{1}{c}{ Name } & \multicolumn{1}{c}{ Protein structure } & Author/refs. \\
\hline 242 & bsHN-CD3 & Flag-VH-L-VL-L-VH-VL-His & Haas et al (10) \\
290 & bsHN-CD28 & Flag-VH-L-VL-L-VH-VL-Etag & Haas et al (10) \\
769 & bsFo-CD3 & Flag-VH-L-VL-L-VH-VL-His & This study \\
337 & bsFo-CD28 & Flag-VH-L-VL-L-VH-VL-Etag & This study \\
\hline
\end{tabular}

aThe protein no. refers to the plasmid that encodes for a particular protein. BAb constructs contained scFv against viral surface antigens (HN and F) and against the CD3 and CD28 molecules. The upper two proteins bind to the viral HN molecule (no. 242: bsHN-CD3 and no. 290: bsHN-CD28), the lower two to F (no. 769: bsF-CD3 and no. 337: bsF-CD28). The N- and C-terminal Tag sequences, Flag tag or His 6 -tag, were used, respectively, for the detection in Western blot or FACS and for the purification of the proteins.

previously described HN-specific fusion protein. This is particularly true for the protein no. 769 (bsF-CD3) since no protein could be detected by Coomassie staining (Fig. 2B). Nevertheless, a specific band corresponding to the expected size of the protein was detectable in Western blot within the elution fraction obtained with $200 \mathrm{mM}$ imidazole (Fig. 2C).

The obtained bsAb proteins were further characterized for their binding properties. For this purpose, the produced proteins were analyzed by FACS since an essential prerequisite for the functionality of proteins is their binding specificity. To this end, we used several established cell lines including Ltk-transfectant cells expressing one of the two viral antigens or both simultaneously. In addition, we used the T-cell lymphoma line JurkatCD28 (JCD28), which expresses the CD28 but also the CD3 molecule at its surface.

The results of the binding assays for $\mathrm{HN}$ and F-specific proteins are shown in Fig. 2D. The HN constructs bound to Ltk-HN cells but not to Ltk-F, while the F-specific proteins bound to Ltk-F but not to Ltk-HN cells (Fig. 2D). All the four proteins tested bound to the Ltk- $(\mathrm{HN}+\mathrm{F})$ cells and to JCD28 cells (Fig. 2D). They also all bound to NDV-infected tumor vaccine cells (data not shown).

The proteins were further characterized by quantifying their binding activity to the viral surface proteins $\mathrm{HN}$ and $\mathrm{F}$ expressed at the surface of the Ltk-(HN+F). The dilution at which just $50 \%$ of the cells were stained, was given an arbitrary value of $10,000 \mathrm{U} / \mathrm{ml}$. This could be compared to the total protein concentration determined by the Bio-Rad method to obtain specific $\mathrm{HN}$ - or F-associated activity in $\mathrm{U} / \mu \mathrm{g}$. The proteins no. 337 and 769 contained 336.30 and $330.20 \mathrm{U} / \mu \mathrm{g}$ respectively, much less than proteins no. 242 and 290 (Table I).

Analysis of signal 1 induced by bsF-CD3. To determine the functional activity of bsF-CD3 (no. 769), this protein was titrated in a $\mathrm{T}$ cell proliferation assay based on Ltk-F cells as stimulator cells. The amount of recombinant protein added varied from $5 \mathrm{ng} /$ well to $100 \mathrm{pg} /$ well (Fig. 3A). For comparison, the protein bsHN-CD3 (no. 242) was titrated in parallel using Ltk-HN as stimulator cells. The construct bsF-CD3 had a much lower activation potential than the protein bsHNCD3 (Fig. 3A). It lost nearly its entire effectiveness within the narrow concentration range between 1-5 ng/well. In contrast, the protein bsHN-CD3 (242) showed its proliferation inducing potential across a larger range (down to $10 \mathrm{pg} / \mathrm{well}$ ). Similar results were obtained in proliferation assays performed with Ltk $(\mathrm{HN}+\mathrm{F})$ cells (data not shown). A sub-optimal concentration of bsF-CD3 required for co-stimulation assays, was determined to be in the range of 0.2-1 ng/well, depending on the donor of the T cells.

The functional specificity of the protein bsF-CD3 was also demonstrated. For this purpose, bsF-CD3 (5 ng/well) was given to $1 \times 10^{4}$ irradiated Ltk-F or Ltk-HN cells in a 96-well plate. Then $1 \times 10^{5}$ purified responder $\mathrm{T}$ cells were added per well. In other wells, bsF-CD3 was replaced by an optimal dose of bsHN-CD3 (10 ng/well). After 48 h, 1 mCi ${ }^{3} \mathrm{H}$-labeled thymidine was added to the wells. The cells were harvested after a further $18 \mathrm{~h}$ to measure the incorporated radioactivity. The protein bsF-CD3 stimulated human T cells only in the presence of Ltk-F cells (Fig. 3B). In contrast, the protein bsHN-CD3 stimulated T cell proliferation only with Ltk-HN cells (Fig. 3B). The controls (T cells alone, with only bsF$\mathrm{CD} 3$ or with only the stimulator cells) did not show any $\mathrm{T}$ cell proliferation (Fig. 3B). In order to substantiate the need for cross-linking, a blocking mAb was added at a final concentration of $5 \mu \mathrm{g} / \mathrm{ml}$. As expected, only the anti-F antibody, and not the anti-HN mAb, blocked the $\mathrm{T}$ cell proliferation induced by Ltk-F cells as stimulators and bsF-CD3 protein as crosslinking agent (Fig. 3C). The results of Fig. 3B and C demonstrate functional activity and specificity of bsF-CD3.

Analysis of signal 2 induced by bsF-CD28 and absence of competition by bsHN-CD3. To analyze the co-stimulatory potential of bsF-CD28 (no. 337), we studied first the binding of this molecule to NDV-infected cells. Ltk-Wt cells were infected overnight with 100 HU NDV Ulster before incubating them with varying amounts of protein bsF-CD28 (no. 337) in the absence or presence of excess amounts of the protein bsHN-CD3 (no. 242). The binding of the bsF-CD28 was evaluated by flow cytometry using anti-E-tag primary antibody (mouse IgG1 isotype), a biotinylated goat anti-mouse IgG1 isotype-specific secondary antibody and finally a streptavidinPE conjugate. The FACS analysis (Fig. 4A) revealed dosedependent binding of the protein bsF-CD28 (no. 337) to NDV-modified cells (compare red curves to controls; gray). As expected, this binding was not competed by an excess of the protein bsHN-CD3 when bound to $\mathrm{HN}$ at the same cell surface. 
A)

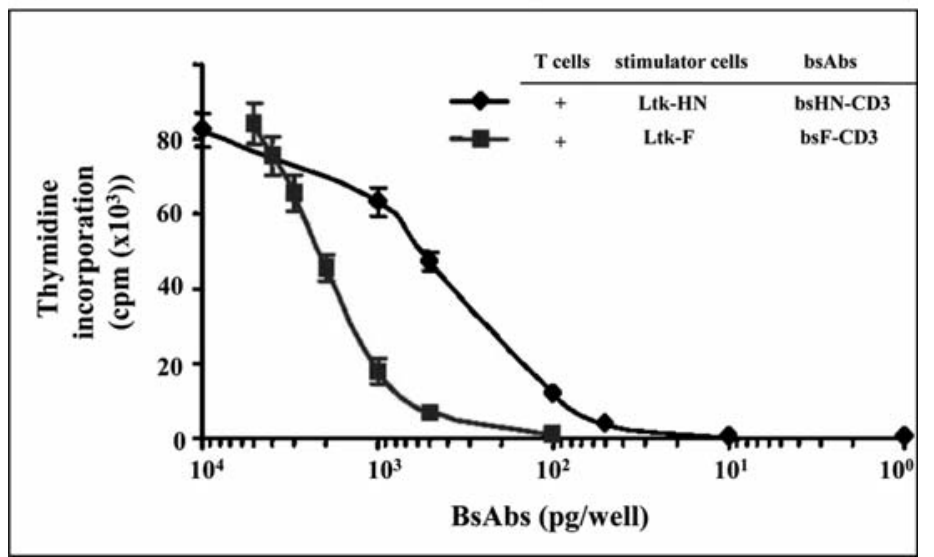

B)

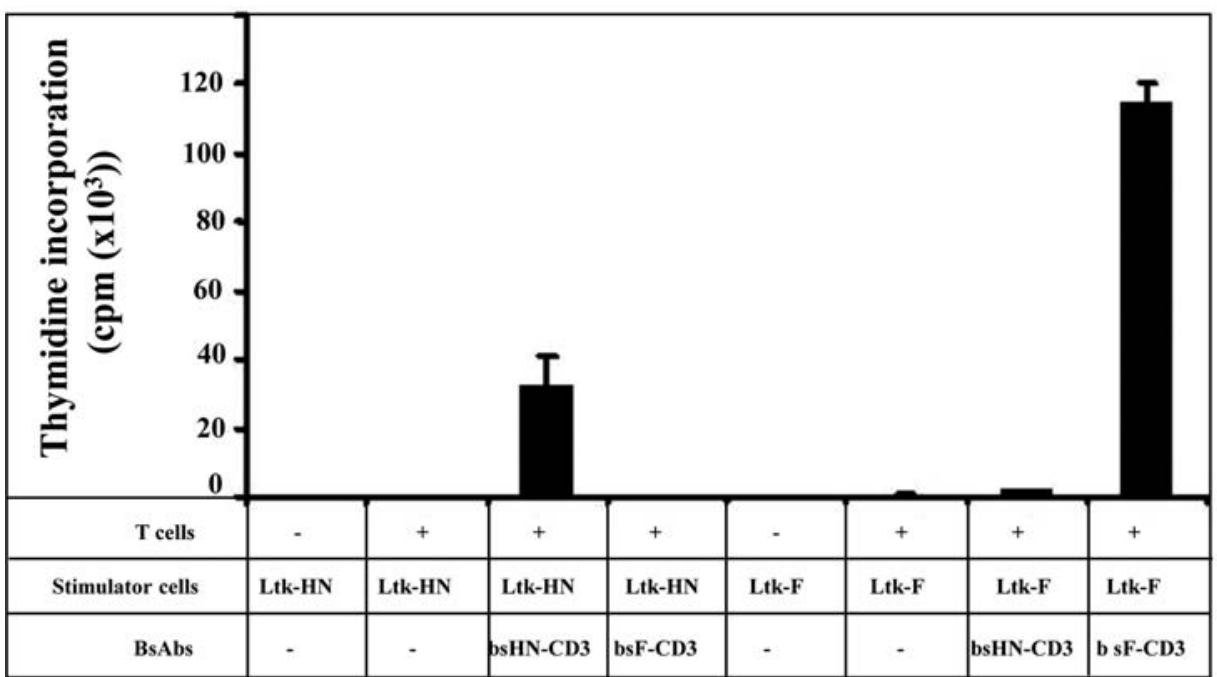

C)

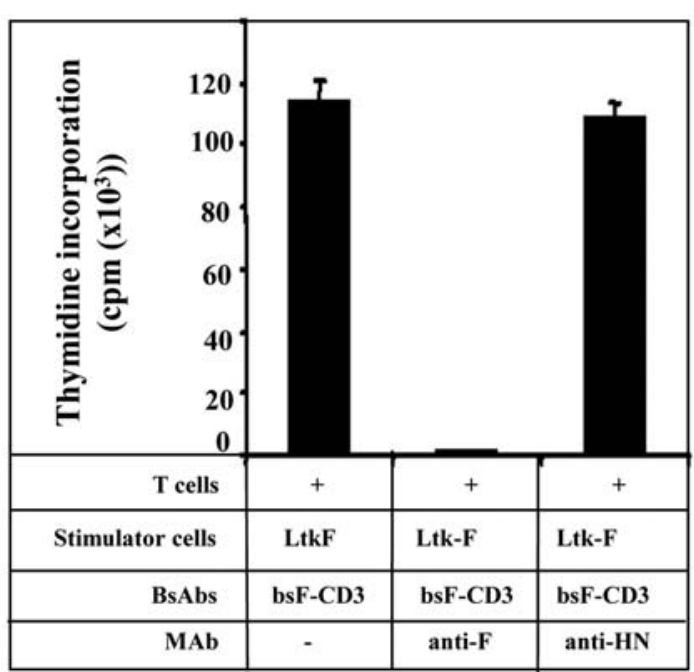

Figure 3. Functional activity and specificity of bsF-CD3. (A) Titration of the bsHN-CD28 and bsHN-CD3 in a T cell proliferation assay. Purified T cells $\left(1 \times 10^{5}\right.$ per well) were incubated for two days with $1 \times 10^{4}$ irradiated Ltk-HN or Ltk-F cells in the presence of different amounts of the two purified bsAbs bsHN-CD3 (black curve) or bsF-CD3 (red curve). After addition of $1 \mathrm{mCi} 3 \mathrm{H}$-labeled thymidine per well, the cells were incubated for another $18 \mathrm{~h}$ before being harvested on a filter. The incorporation of radioactivity as indicator of $\mathrm{T}$ cell proliferation was measured using a liquid scintillation counter. All values were determined in triplicate and are expressed as mean \pm standard deviation. (B) Specificity of bsHN-CD3 (no. 242) and bsF-CD3 (no. 769 ) in T cell stimulation assays. Purified human T cells $\left(1 \times 10^{5}\right.$ per well) were incubated for two days with $1 \times 10^{4}$ irradiated Ltk-HN or Ltk-F cells with $10 \mathrm{ng} / \mathrm{well}$ of purified bsHN-CD3 (no. 242) or $5 \mathrm{ng} /$ well of purified bsF-CD3 (no. 769). Afterwards, T cell proliferation was assessed as in (A). All values were determined in triplicate and are expressed as mean \pm standard deviation. $\mathrm{T}$ cells on stimulator cells alone or with stimulator $\mathrm{T}$ cells alone without bsAbs served as negative control. (C) Specific blocking of Ltk-F+bsF-CD3 induced T cell proliferation by F- (but not HN-) specific mAb. Purified T cells (1x105 per well) were incubated with $1 \times 10^{4}$ irradiated Ltk-HN or Ltk-F cells in the presence of bsF-CD3 (5 ng/well). MAbs specific for HN and F were added at a final concentration of $5 \mu \mathrm{g} / \mathrm{ml}$ according to the table underneath the figure. T cell proliferation was assessed as described in (A). All values were determined in triplicate and are expressed as mean \pm standard deviation. 
A)

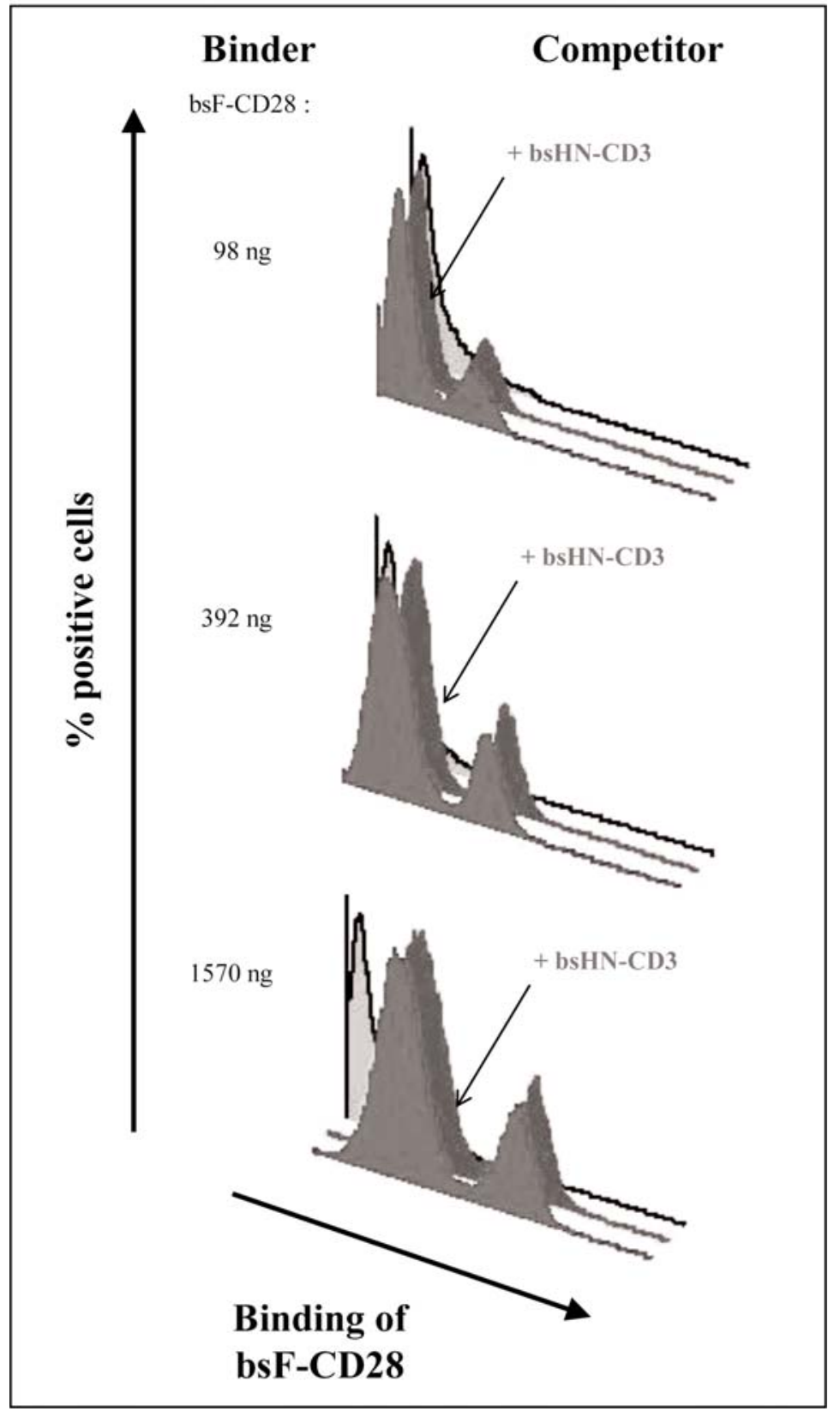

Figure 4. Strong binding and co-stimulatory activity of bsF-CD28 in combination with bsHN-CD3. (A) Absence of competition between bsHN-CD3 and bsFCD28 for binding to the stimulator cells. Ltk cells $\left(1 \times 10^{5}\right)$ which were infected overnight with NDV (100 HU/10 7 cells) were incubated with different amounts of bsF-CD28 (98, 392 and $1570 \mathrm{ng}$ ) in the absence or in the presence of $540 \mathrm{ng}$ of the competitive protein bsHN-CD3 (no. 242). As a negative control, NDV-modified Ltk cells were incubated with only $540 \mathrm{ng}$ of protein no. 242 (gray). The cells were stained in a first step with an anti-E-tag mAb (or a mouse IgG1 isotype as negative control), then with a biotinylated goat antibody specific for mouse IgG1 and finally with a streptavidin-PE conjugate before being analyzed by flow cytometry. Dead cells were excluded by staining with propidium iodide.

We then analyzed the CD28-activity of bsF-CD28 (337) in a $\mathrm{T}$ cell proliferation assay based on the use of Ltk-NDV cells as stimulator cells. We loaded these cells with suboptimal concentrations of bsHN-CD3 in the range of 150-50 pg/well (signal 1). Upon addition of bsF-CD28 (132 U/well), we saw a significant increase of thymidine uptake in comparison to the controls which received only signal 1 . BsF-CD28 alone with Ltk-NDV cells, as expected, did not induce T cell proliferation.

Competition between bsHN-CD3 and bsHN-CD28 for binding to $H N$ of stimulator cells. Binding competition between bsHN-CD3 and bsHN-CD28 to $\mathrm{HN}$ expressed at the cell surface of a tumor vaccine was tested as follows. $\mathrm{Ltk}(\mathrm{HN}+\mathrm{F})$ cells were incubated with various combinations of both bsAbs. The binding of individual bsAbs was then determined by flow cytometry using anti-His (mouse IgG2a) and anti-Etag (mouse IgG1) primary antibodies and isotypespecific fluorescence-labeled secondary antibodies.

The binding of bsHN-CD3 (evaluated as a percentage of stained cells) was compared in presence or absence of the competing recombinant protein bsHN-CD28 (125 ng, Fig. 5Aa). Conversely, the binding of bsHN-CD28 protein in 
B)

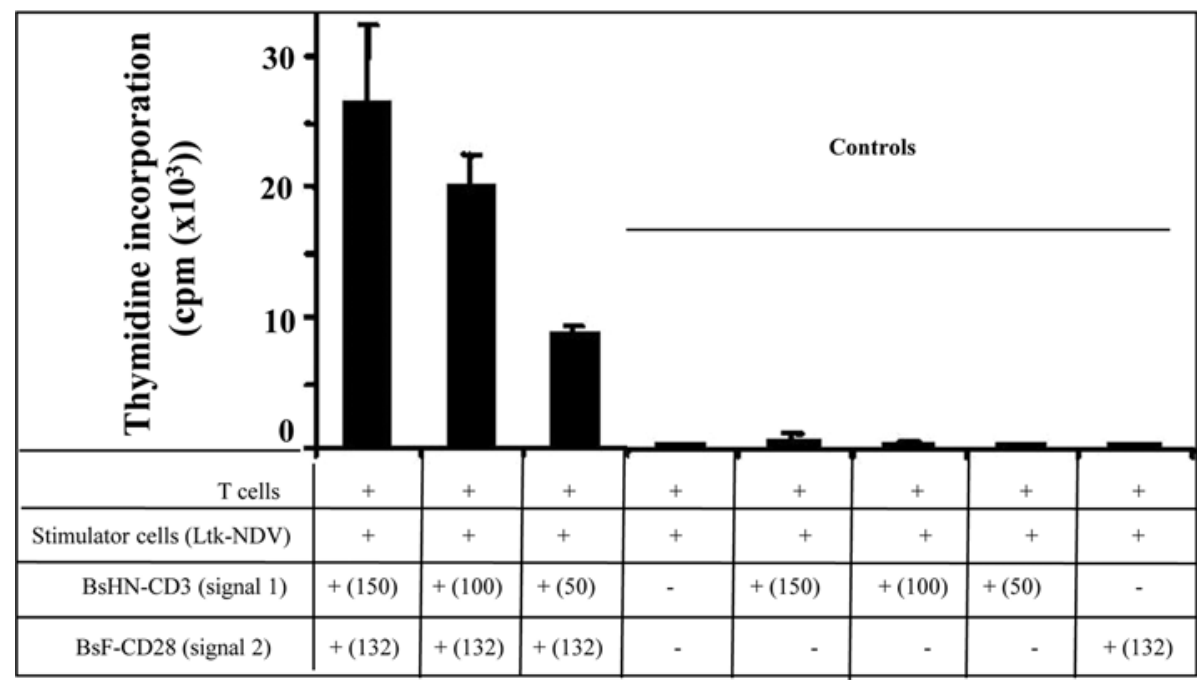

Figure 4. Continued. (B) Strong T cell proliferation induced by combination of bsF-CD28 and bsHN-CD3. Purified T cells (1x10 $5 /$ well) were incubated with $1 \times 10^{4}$ irradiated Ltk cells which were modified beforehand through addition of NDV (100 HU/10 ${ }^{7}$ cells). To this co-culture were added bsHN-CD3 (at suboptimal concentrations: 150,100 and $50 \mathrm{pg} /$ well) and bsF-CD28 at a concentration of $131.95 \mathrm{U} /$ well. Two days later, $1 \mathrm{mCi}{ }^{3} \mathrm{H}-\mathrm{labeled}$ thymidine per well was added and further incubated for $18 \mathrm{~h}$. Incorporated radioactivity was then measured by a liquid scintillation counter. All values were determined in triplicate and are indicated as mean \pm standard deviation. T cells and stimulator cells without any bsAbs or with bsAbs, added individually, served as controls.

presence or absence of competing bsHN-CD3 protein $(250 \mathrm{ng})$ was analyzed (Fig. 5Ac). It can be seen that $125 \mathrm{ng}$ of bsHNCD28 reduced the binding of bsHN-CD3 over a broad dose range. bsHN-CD3 (250 ng) reduced the binding of bsHNCD28 only in the lower dose range of 30-2 ng. The density of the respective constructs bound to the surface of Ltk- $(\mathrm{HN}+\mathrm{F})$ cells was also measured and is expressed as mean fluorescence intensity (mean AFU) in Fig. 5Ab and d. The results show that the two constructs bind best when they are tested alone, without any competition between them. The fact that the competitive effect of protein bsHN-CD28 on the protein bsHN-CD3 is less visible than in the reverse situation, is due to the above described staining of construct bsHN-CD28 through the biotin/streptavidin-mediated amplification system. In any case, the analysis of the surface density of bound proteins confirms the effect of competition: without the protein bsHN-CD28, $250 \mathrm{ng}$ of the protein bsHN-CD3 gave a mean fluorescence intensity of 30. In the presence of $125 \mathrm{ng}$ of the protein bsHN-CD28, the mean value was reduced to 16.5 (Fig. 5Ab). Conversely, the mean values increased with $250 \mathrm{ng}$ bsHN-CD3 (242), when the concentration of bsHNCD28 decreased (Fig. 5Ad).

Analysis of $T$ cell activation through stimulator cells expressing CD3- and CD28-specific constructs separately bound to either $H N$ or $F$. We analyzed in functional assays the implications of potential competition between proteins for introducing signal 1 and 2. Purified T cells were incubated with suboptimal doses of anti-CD3 (5 pg/well for bsHN-CD3 or $500 \mathrm{pg} /$ well for bsF-CD3) and with Ltk-(HN+F) cells (which were $\gamma$-irradiated with $100 \mathrm{~Gy}$ ) as stimulator cells. This condition leads to only marginal thymidine uptake (Fig. 5B, T cells and stimulator cells with CD3 signal alone).
The addition of an excess of bsHN-CD28 (210 ng/well) as a co-stimulatory signal when used in combination with the protein bsHN-CD3 led to a weak, albeit significant $\mathrm{T}$ cell proliferation in comparison to the above-mentioned control (Fig. 5B, p<0.041). The presence of large amounts of bsHNCD28 likely suppressed most of the other HN-specific protein from its binding sites. When bsHN-CD3 was replaced by bsF-CD3, an equivalent amount of the F-binding and CD3 triggering protein, a strong $\mathrm{T}$ cell proliferation was observed (Fig. 5B, p<0.000009). In this case, the 2 signal inducing proteins could bind optimally to the stimulator cells without interfering with each other.

Analysis of the effect of providing the co-stimulatory signal on a third-party cell. To avoid an interference between the bsAbs having specificity for the same viral antigen, these proteins could be loaded onto different vaccine cells. That would mean that effector T cells must be in contact with both vaccine cell types which provide signal 1 and 2 . To test this situation, purified $\mathrm{T}$ cells were incubated with three different combinations of Ltk stimulator cells loaded with bispecific proteins (Fig. 6).

The first combination which caused $\mathrm{T}$ cell activation consisted of both signals (CD3 and CD28) in the form of $\mathrm{HN}$-specific proteins added to a mix composed of $50 \%$ Ltk$\mathrm{HN}$ and $50 \%$ Ltk-F cells. In the second combination which did not cause $\mathrm{T}$ cell activation, the protein bsHN-CD3 was replaced by an equivalent amount of bsF-CD3 bound to Ltk-F within the cell mix Ltk-HN + Ltk-F. In the third combination which caused $\mathrm{T}$ cell activation, the mix of Ltk cells was replaced by Ltk cells modified with $100 \mathrm{HU}$ NDV so that the two proteins bsHN-CD3 and bsF-CD28 were bound to the same cell, albeit to different antigens. Thus, when both 
A)
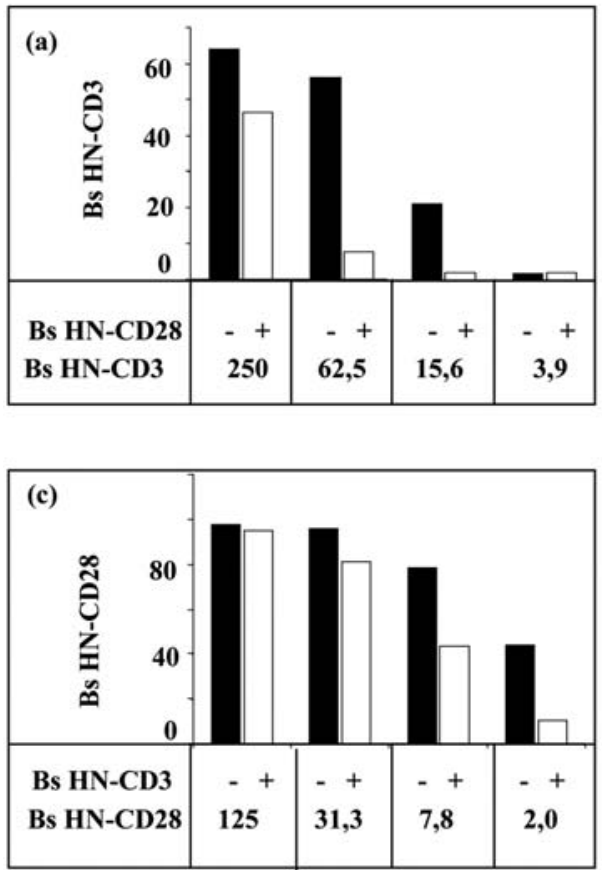

Mean (AFU)

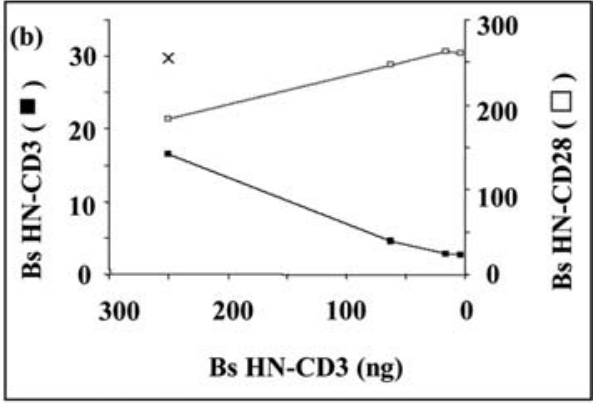

B)

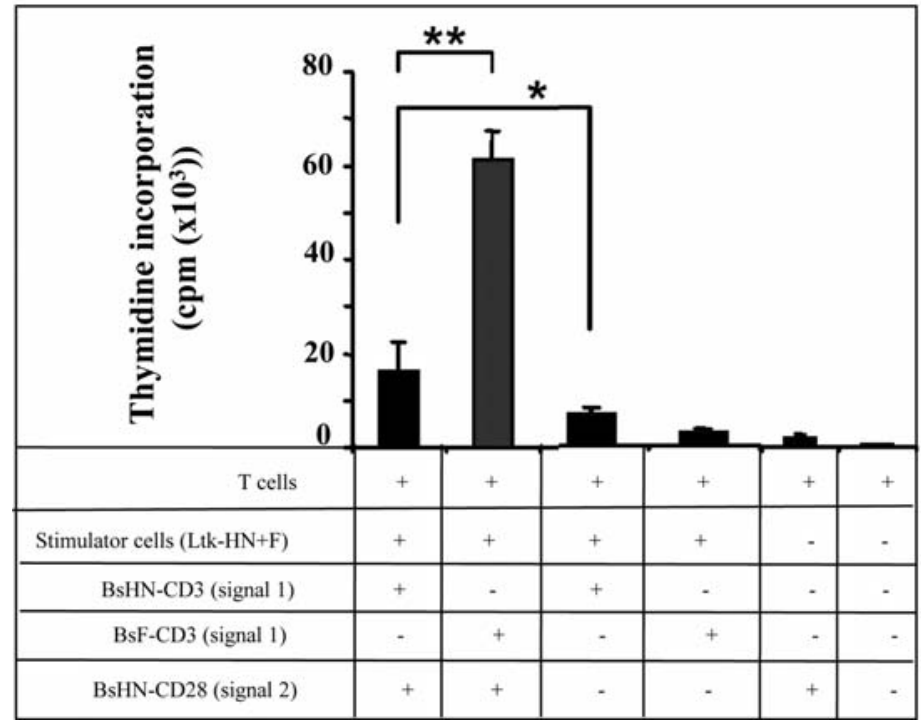

Figure 5. Reduced binding and functional activity through competition between the two T cell stimulating bsAbs when binding occurred to the same viral anchor molecule (HN). (A) Reducing binding through competition between bsHN-CD3 and bsHN-CD28 for HN. (a and b) Ltk(HN+F) cells (1x105) were incubated with the indicated amounts of protein bsHN-CD3. This was done in the presence or absence of 125 ng protein no. 290 . The BsAbs were specifically detected by anti-His (mouse IgG2a isotype) or anti-E-Tag (mouse IgG1 isotype) primary antibody. After a staining with FITC-conjugated goat anti-mouse $\mathrm{IgG} 2 \mathrm{a}$ isotype-specific or biotinylated goat anti-mouse IgG1 isotype-specific secondary antibody, an incubation was carried out with PE-conjugated streptavidin. The analysis was performed by flow cytometry. The bars in (a) show the percentage of positively stained cells, the value in (b) corresponds to the mean fluore-scence intensity measured in these assays. (c and d) Conversely but in an analogous way, the binding of bsHN-CD28 (added at different amounts from 2 to $125 \mathrm{ng}$ ) in the presence or absence of the protein bsHN-CD3 (250 ng/well). The bars in (c) show the \% positive cells, the values in (d) show the mean fluorescence intensity. The crosses in (b) and (d) show the mean binding of the examined protein [bsHN-CD28(b) or bsHN-CD3(d)] without any competition. Dead cells were excluded by PI staining. (B) Co-stimulation with bsHN-CD28 protein under competing and non-competing conditions. Purified naïve $\mathrm{T}$ cells $\left(1 \times 10^{5}\right)$ were incubated for two days with $1 \times 10^{4}$ irradiated $\mathrm{Ltk}(\mathrm{HN}+\mathrm{F})$ cells and a suboptimal concentration of bsHN-CD3 (5 pg/well) or bsF-CD3 $(500 \mathrm{pg} / \mathrm{well})$ and an excess of the co-stimulatory molecule bsHN-CD28 (210 ng/well). After addition of $1 \mathrm{mCi}{ }^{3} \mathrm{H}$-thymidine per well and incubation for another $18 \mathrm{~h}$, the cells were harvested and the radioactivity incorporated was measured in a liquid scintillation counter. All values were determined 5-fold and expressed as mean \pm standard deviation. As controls, served either T cells alone or mixtures of $\mathrm{T}$ cells with stimulator cells in the presence of only one bsAb. ${ }^{*} \mathrm{p}<0.041,{ }^{* *} \mathrm{p}<0.000009$.

signals were separated on different cells (combination 2 with Ltk-F + bsF-CD3 and Ltk-HN + bsHN-CD28), there was no capacity of this mixture to stimulate $\mathrm{T}$ cells anymore. These results demonstrate that, for optimal activation, coupling of 


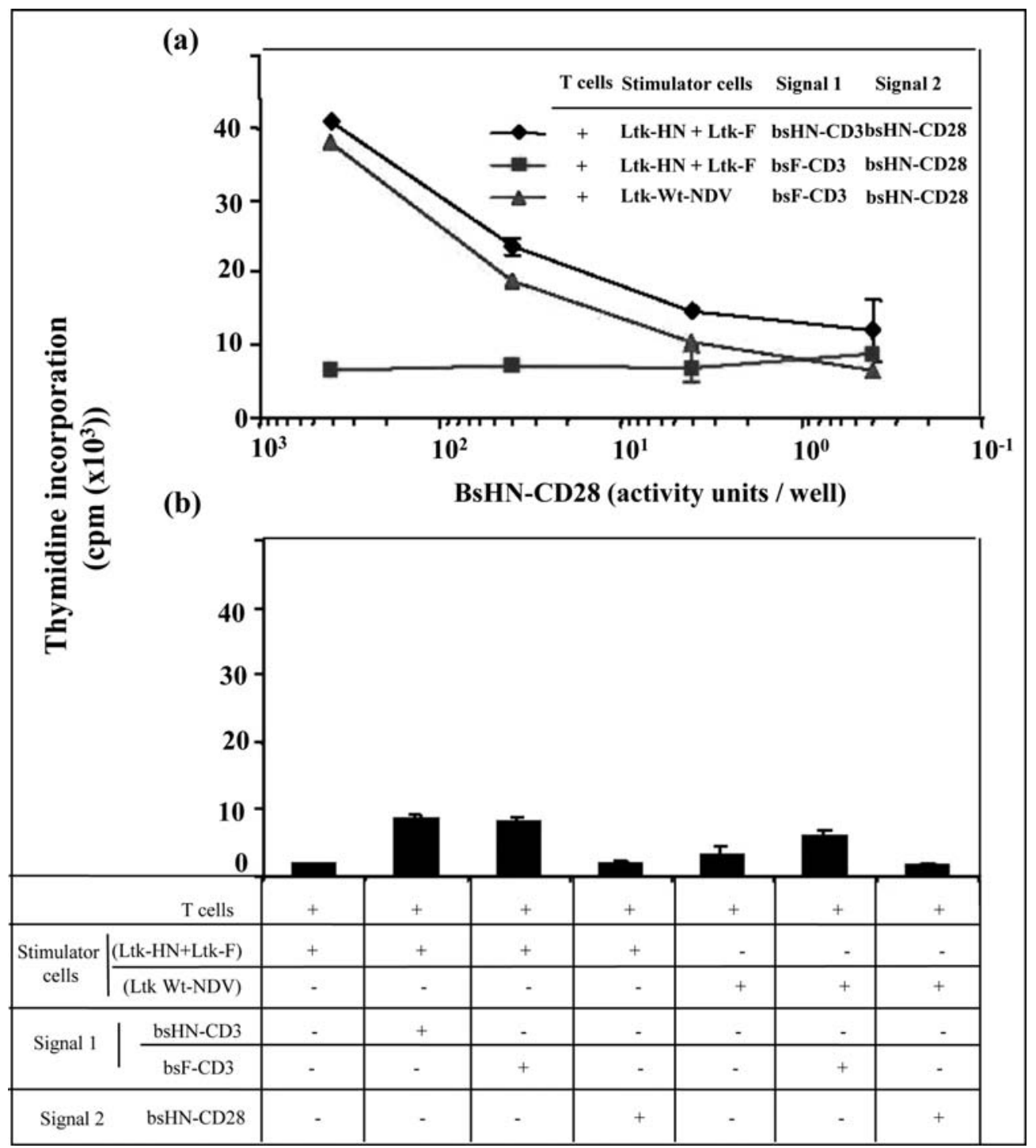

Figure 6. Testing of signal combinations on one presenting cell versus two different presenting cells. Purified naive T cells $\left(1 \times 10^{5}\right)$ were incubated with $2 \times 10^{4}$ irradiated Ltk cell mix (consisting in equal parts of Ltk-HN and Ltk-F cells) or with $2 \times 10^{4}$ irradiated Ltk modified beforehand through the addition of $100 \mathrm{HU}$ NDV and $1 \mathrm{~h}$ incubation. The different bsAbs, bsHN-CD3 (50 pg/well) or to bsF-CD3 (1.6 ng/well) and varying amounts of bsHN-CD28, were added. Two days later, addition of $1 \mathrm{mCi}{ }^{3} \mathrm{H}$-labeled thymidine per well and a further incubation for another $18 \mathrm{~h}$ allowed the quantification with a liquid scintillation counter of the $\mathrm{T}$ cell proliferation induced. All values were determined in triplicate and are expressed as mean \pm standard deviation. (A) Results from experimental groups; (B) results from controls.

the two fusion proteins to the same vaccine cell is advantageous. This warrants that both signals can be co-delivered simultaneously to the effector cells.

Collectively, the results presented in this study show that, for an ideal tumor vaccine, the bsAbs proteins should be bound to the same tumor vaccine cell. In the case of the ATV-NDV vaccine, the recombinant molecules can be bound separately to the two viral proteins $\mathrm{HN}$ and $\mathrm{F}$. This is not necessarily required always, but it needs to be considered under particular circumstances to prevent competition for binding.

\section{Discussion}

In this study, novel bispecific antibodies were constructed and tested for their capacity to improve $\mathrm{T}$ cell stimulation by the ATV-NDV tumor vaccine that was developed over the last 10 years. The two previously described bispecific constructs bsHN-CD3 and bsHN-CD28 bind with one arm to the viral HN molecule at the surface of the vaccine cells. The second arm is directed either against the T-cell receptor (TCR)-associated CD3 molecule, or against the co-stimulatory CD28 receptor molecule $(10,12)$. These two signals are expected to recruit and activate naïve $\mathrm{T}$ cells at the site of the administered vaccine while the original ATV-NDV vaccine works primilarly on the basis of re-activation of tumorreactive memory $\mathrm{T}$ cells (13). Bispecific antibodies binding to the F protein of NDV are presented in this study for the first time. Through the addition of bispecific molecules binding at the surface of the tumor vaccine either to $\mathrm{HN}$ or $\mathrm{F}$, the anti-tumor effect of the tumor vaccine is expected to be considerably enhanced.

The key point of this study was to increase the effectiveness of the pre-existing and previously described ATV-NDV 


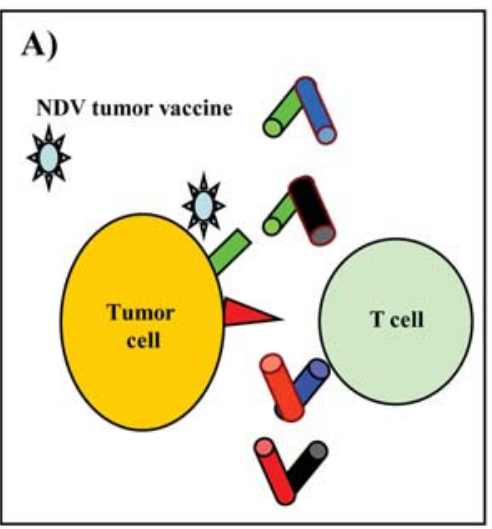

B)

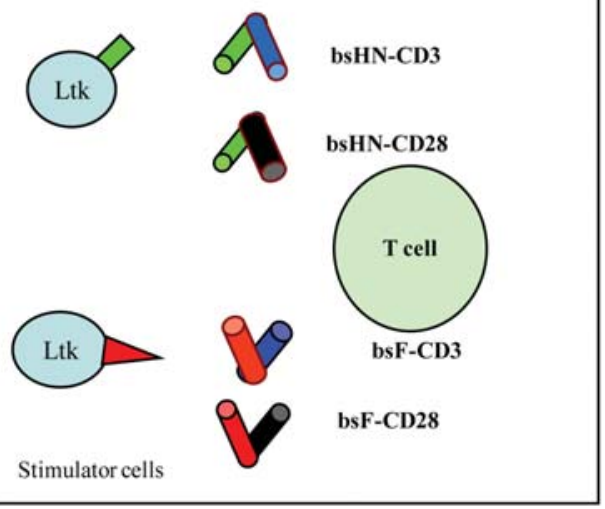

C)

HN- and F-binding ligands

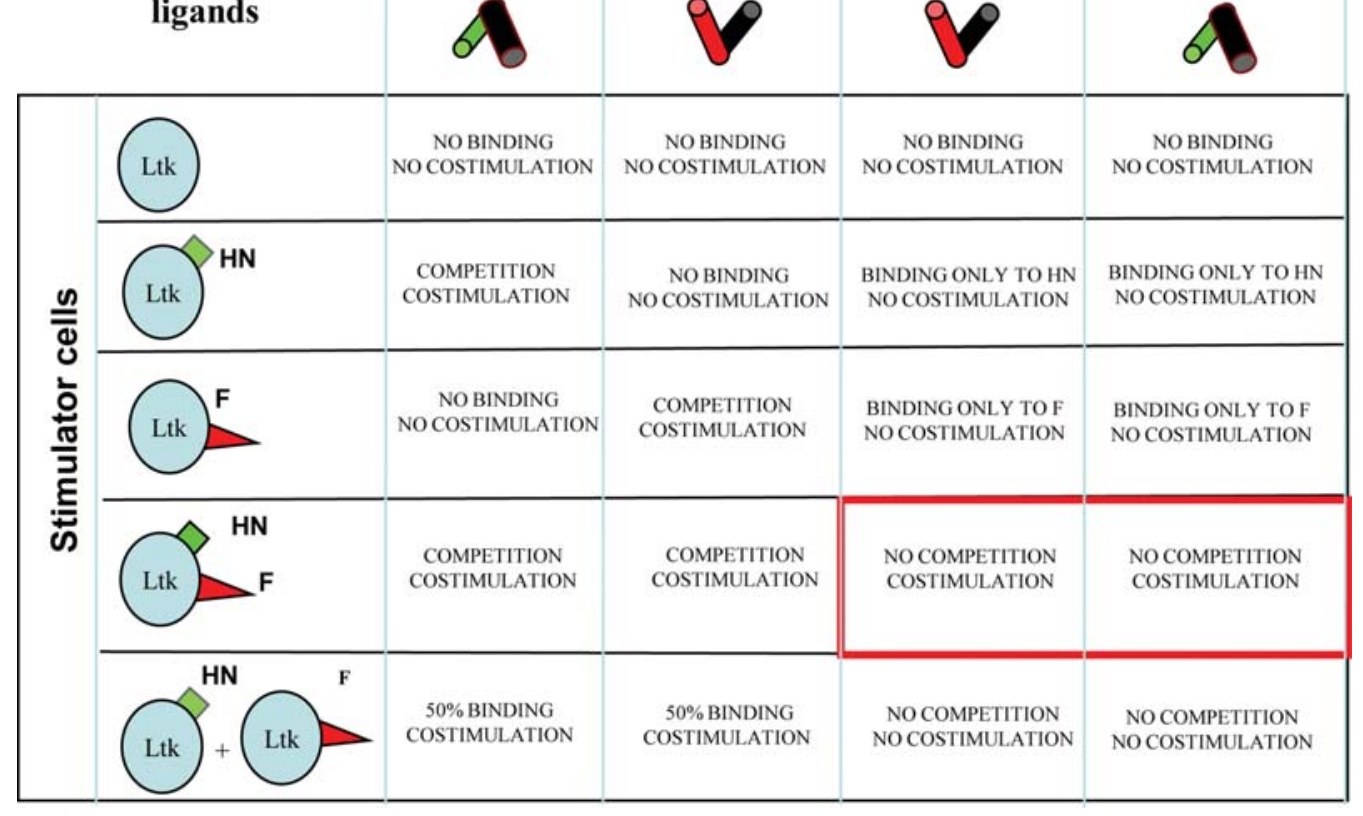

Figure 7. Cartoon of T cell co-stimulation using 4 bsAbs bound in various ways to stimulator cells. (A) Schematic presentation of the tumor vaccine ATVNDV-bAbs. The ATV tumor vaccine is obtained by $\gamma$-irradiation and infection by NDV of tumor cells. This vaccine is then incubated with bsAbs which add additional signals at the surface of the vaccine. Incubation of such an optimized tumor vaccine with T cells leads to their proliferation. (B) Ltk transfectants as stimulator cells. The use of transfectants which express the viral HN or F protein of NDV can be used as model for the binding of the different bsAbs introducing signals 1 and 2. Such a system can also be used in T cell proliferation assays. (C) Combinations of stimulator cells with bAbs. The table summarizes the results of co-culture of T cells with stimulator cells expressing different combinations of viral antigens and loaded with various combinations of bAbs binding to the 2-viral anchoring molecules $\mathrm{HN}$ and F. The optimal conditions, under which co-stimulation is obtained without competition, are highlighted.

tumor vaccine by coupling immunostimulatory proteins. For solving the mechanistic questions that are related to an optimal loading of the vaccine cells with the constructs, 2 aspects had to be clarified: i) Is the utilization of 2 competing proteins bound to a common anchor molecule of the vaccine suitable or critical? ii) Would it be superior if each vaccine cell would expose the two bsAbs attached to two separate viral anchoring molecules? iii) Would it be possible to attach the two bsAbs delivering different signals to separate subpopulations of the vaccine cells?

To be able to answer these specific questions, it was important to have transfectants which express one or even both of the viral surface proteins of NDV. Such transfectants, which were successfully established, present certain advantages when compared to the classical NDV-infected tumor vaccines. First, the viral antigens are presented in a homogeneous manner on their surface. Secondly, virusinduced additional effects (as for example the production of interferon- $\alpha$ by the vaccine cells through viral replication) are avoided.

For the elaboration of bispecific fusion proteins, we used the recombinant antibody technology: first, we generated single chain $(\mathrm{scFv})$ antibodies in which the variable regions of heavy and light chains are covalently combined via a linker sequence. We then fused the respective specificities and expressed them in $\mathrm{CHO}$ cells. The molecular weights of the new fusion proteins corresponded to the ones described for the HN-binding constructs (10). 
The reason for the choice of these two signals is the now generally accepted paradigm that the control or elimination of cancer is caused primarily by $\mathrm{CD}^{+}$cytotoxic $\mathrm{T}$ cells (CTL) (15). CD4+ helper $\mathrm{T}$ cells enhance the CTL response and also contribute to the production of TAA-specific antibodies by B cells (15). It has been reported, however, that tumors may develop immune escape mechanisms through which they avoid actively the surveillance and their elimination by the immune system (e.g. by a reduced processing of the peptides, by reduced presentation of MHC molecules or by down-regulation of co-stimulatory molecules) $(4,16)$.

An advantage of our bsAbs, which contain CD3 binding properties, is that even when they are expressed on immunoresistant tumor cells, they can still activate $\mathrm{T}$ cells since tumor-associated MHC molecules or co-stimulatory molecules are not required. Other groups working with bispecific antibodies have developed reagents of the type 'CD3xTumor' antigen or 'CD28xTumor antigen' $(17,18)$. These reagents targeting tumor antigens are much more restricted with regard to application compared to our reagents targeting viral antigens which can be easily introduced into any tumor cell through simple infection.

Unfortunately, the F-specific fusion proteins were produced at smaller quantities than their HN-specific counterparts. A possible reason may be an intrinsic toxicity of F-specific $\mathrm{scFv}$, a feature that was described for certain fusion proteins when expressed both in prokaryotic and eukaryotic cells $(19,20)$. Also, a position effect and the associated correct folding may play an important role. For some of our constructs an almost complete loss of binding capability in FACS analysis was observed when the individual components (i.e., the $\mathrm{scFv}$ fragments) of antibodies were reversed in their position (data not shown). For the four fusion proteins described, our data overall suggest that the individual components of the fusion proteins were correctly folded during their synthesis, without affecting each other negatively. In this respect, the linker (Gly4Ser1), consisting of 4 glycines and a serine allowed to connect the individual components by allowing maximum flexibility. Potential steric inhibitory effects between the scFvs in binding to two different targets are thereby avoided $(21,22)$. The 4 bsAbs produced showed in FACS analysis all the expected binding specificities. HNspecific constructs bound only to Ltk-HN and Ltk- $(\mathrm{HN}+\mathrm{F})$ cells. In contrast, F-specific fusion proteins bound specifically to Ltk-F cells and of course also to Ltk-(HN+F) cells.

It was also important that the constructs were not only able to bind to defined cell lines, but also to NDV-infected human tumor cells. This was verified with the MCF-7 breast cancer line and with other human tumor cells (data not shown). Anti-CD3 and anti-CD28 binding was demonstrated with JCD28 leukemia cells and by the fact that naïve T cells could become activated in spite of the fact that binding signals were undetectable by FACS analysis (data not shown).

Following the concept of the use of bispecific singlechain antibodies $(18,23,24)$, T cells can be activated only if the targeted receptor proteins (e.g. CD3 and CD28) become cross-linked. Accordingly, the monovalent bispecific fusion proteins in solution were not capable to activate T cells. Only when bound to NDV at the surface of stimulator cells were they capable of cross-linking and $\mathrm{T}$ cell activation.
In the presence of a sub-optimal CD3-signal, the addition of fusion proteins, which contain anti-CD28 scFv, significantly increased $\mathrm{T}$ cell-proliferation (co-stimulation). However, this was the case only under the condition that both proteins were linked to the same stimulator cells. Similar observations were reported for other systems (25-27). Also, for specific antigenpresenting cells, signals 1 and 2 have to be expressed on the same cell in order to achieve optimal effects $(28,29)$. Studies with fluorescent CD3 and CD28 molecules showed that in $\mathrm{T}$ cells the CD28 molecule co-localizes with the TCR/CD3complex (30).

It makes sense that effector $\mathrm{T}$ cells are activated much more efficiently if the various stimuli are presented together and not via separate cells. This is likely the most appropriate physiological context that allows $\mathrm{T}$ cells to receive the signals in an integrative fashion through an immunological synapse (31). Fig. 7 presents a cartoon of the various possible combinations of bsAbs when attached to NDV-infected tumor vaccine (A) or to Ltk transfectants (B and C). This figure summarizes our findings and provides answers to the questions of this discussion. Optimal conditions for co-stimulation under non-competing conditions of binding of the two fusion proteins are highlighted. If the two fusion proteins bind to the same viral anchor molecule, this study has shown that competition can occur so that $\mathrm{T}$ cell activation is not optimal.

In summary, the data presented here demonstrate which are the optimal conditions for coupling two immunostimulatory fusion proteins to viral anchor molecules of the tumor vaccine ATV-NDV. This allows to strengthen signal 1 induced by TAA via additional CD3 stimulation and signal 2 induced by $\mathrm{HN}(7,8)$ via additional $\mathrm{CD} 28$ stimulation. The second generation vaccine ATV-NDV+bsCD3/CD28 is possibly superior to beads coated with antiCD3/CD28 because it contains relevant TAAs and upregulated MHC, ICAM-I and LFA-3 (2).

\section{Acknowledgements}

This study was part of the PhD thesis of Maximilian Aigner under co-supervision by Professor Dr Volker Schirrmacher and Dr Philippe Fournier. We are particularly grateful to Annette Arnold for technical assistance, especially for help in the production and purification of the recombinant proteins. The writing of this manuscript would not have been possible without the kind financial support of Dr Kleist Stiftung (Berlin, Germany) and Christian-Berndt Stiftung (Rostock, Germany). We gratefully acknowledge also the support by the IOZK (www.iozk.de) (Cologne, Germany).

\section{References}

1. Gilboa E: The makings of a tumor rejection antigen. Immunity 11: 263-270, 1999

2. Washburn B and Schirrmacher V: Human tumor cell infection by Newcastle Disease Virus leads to upregulation of HLA and cell adhesion molecules and to induction of interferons, chemokines and finally apoptosis. Int J Oncol 21: 85-93, 2002.

3. Schirrmacher V: Immunity and metastasis: in situ activation of protective $\mathrm{T}$ cells by virus modified cancer vaccines. Cancer Surv 13: 129-154, 1992.

4. Hicklin DJ, Marincola FM and Ferrone S: HLA class I antigen downregulation in human cancers: T-cell immunotherapy revives an old story. Mol Med Today 5: 178-186, 1999. 
5. Schlom J and Hodge JW: The diversity of T-cell co-stimulation in the induction of antitumor immunity. Immunol Rev 170: 73-84, 1999.

6. Schirrmacher V, Haas C, Bonifer R and Ertel C: Virus potentiation of tumor vaccine $\mathrm{T}$-cell stimulatory capacity requires cell surface binding but not infection. Clin Cancer Res 3: $1135-1148,1997$.

7. Termeer CC, Schirrmacher V, Brocker EB and Becker JC: Newcastle disease virus infection induces B7-1/B7-2-independent T-cell costimulatory activity in human melanoma cells. Cancer Gene Ther 7: 316-323, 2000.

8. Ertel C, Millar NS, Emmerson PT, Schirrmacher V and von Hoegen P: Viral hemagglutinin augments peptide-specific cytotoxic T cell responses. Eur J Immunol 23: 2592-2596, 1993.

9. Schirrmacher V, Haas, C, Bonifer R, Ahlert T, Gerhards R and Ertel C: Human tumor cell modification by virus infection: an efficient and safe way to produce cancer vaccine with pleiotropic immune stimulatory properties when using Newcastle disease virus. Gene Ther 6: 63-73, 1999.

10. Haas C, Lulei M, Fournier P, Arnold A and Schirrmacher V: T-cell triggering by CD3- and CD28-binding molecules linked to a human virus-modified tumor cell vaccine. Vaccine 23 . 2439-2453, 2005.

11. Zeng J, Fournier P and Schirrmacher V: High cell surface expression of Newcastle disease virus proteins via replicon vectors demonstrates syncytia forming activity of $\mathrm{F}$ and fusion promotion activity of HN molecules. Int J Oncol 25: 293-302, 2004.

12. Haas C, Lulei M, Fournier P, Arnold A and Schirrmacher V: A tumor vaccine containing anti-CD3 and anti-CD28 bispecific antibodies triggers strong and durable antitumor activity in human lymphocytes. Int J Cancer 118: 658-667, 2006.

13. Schirrmacher V: Clinical trials of antitumor vaccination with an autologous tumor cell vaccine modified by virus infection: improvement of patient survival based on improved antitumor immune memory. Cancer Immunol Immunother 54: 587-598, 2005.

14. Pardoll D: T cells take aim at cancer. Proc Natl Acad Sci USA 99: 15840-15842, 2002.

15. Romagnani $\mathrm{S}$ : The Th1/Th2 paradigm. Immunol Today 18: 263-266, 1997.

16. Seliger B, Maeurer MJ and Ferrone S: Antigen-processing machinery breakdown and tumor growth. Immunol Today 21: 455-464, 2000

17. Willems A, Schoonooghe S, Eeckhout D, De Jaeger G, Grooten J and Mertens N: CD3 x CD28 cross-interacting bispecific antibodies improve tumor cell dependent T-cell activation. Cancer Immunol Immunother 54: 1059-1071, 2005.

18. da Costa L, Renner C, Hartmann F and Pfreundschuh M: Immune recruitment by bispecific antibodies for the treatment of Hodgkin disease. Cancer Chemother Pharmacol 46 (Suppl.): S33-S36, 2000.
19. Doyle SA: Screening for the expression of soluble recombinant protein in Escherichia coli. Methods Mol Biol 310: 115-121, 2005.

20. Hirano A and Wong TC: Generation of mammalian cells expressing stably measles virus proteins via bicistronic RNA. J Virol Methods 33: 135-147, 1991.

21. Kufer P, Zettl F, Borschert K, Lutterbuse R, Kischel R and Riethmuller G: Minimal costimulatory requirements for $\mathrm{T}$ cell priming and TH1 differentiation: activation of naive human T lymphocytes by tumor cells armed with bifunctional antibody constructs. Cancer Immun 1: 10, 2001.

22. Kufer P, Lutterbuse R and Baeuerle PA: A revival of bispecific antibodies. Trends Biotechnol 22: 238-244, 2004.

23. Hombach A, Tillmann T, Jensen M, Heuser C, Sircar R, Diehl V, Kruis $\mathrm{W}$ and Pohl C: Specific activation of resting $\mathrm{T}$ cells against tumour cells by bispecific antibodies and CD28-mediated costimulation is accompanied by Th1 differentiation and recruitment of MHC-independent cytotoxicity. Clin Exp Immunol 108: 352-357, 1997.

24. Staerz UD, Kanagawa O and Bevan MJ: Hybrid antibodies can target sites for attack by T cells. Nature 314: 628-631, 1985.

25. Biburger M, Weth R and Wels WS: A novel bispecific tetravalent antibody fusion protein to target costimulatory activity for T-cell activation to tumor cells overexpressing ErbB2/ HER2. J Mol Biol 346: 1299-1311, 2005.

26. Li Q, Yu B, Grover AC, Zeng X and Chang AE: Therapeutic effects of tumor reactive $\mathrm{CD}^{+}$cells generated from tumorprimed lymph nodes using anti-CD3/anti-CD28 monoclonal antibodies. J Immunother 25: 304-313, 2002

27. Holliger P, Manzke O, Span M, Hawkins R, Fleischmann B, Qinghua L, Wolf J, Diehl V, Cochet O, Winter G and Bohlen H: Carcinoembryonic antigen (CEA)-specific T-cell activation in colon carcinoma induced by anti-CD3 x anti-CEA bispecific diabodies and B7 $\mathrm{x}$ anti-CEA bispecific fusion proteins. Cancer Res 59: 2909-2916, 1999.

28. Cardoso AA, Seamon MJ, Afonso HM, Ghia P, Boussiotis VA, Freeman GJ, Gribben JG, Sallan SE and Nadler LM: Ex vivo generation of human anti-pre-B leukemia-specific autologous cytolytic T cells. Blood 90: 549-561, 1997.

29. Galvin F, Freeman GJ, Razi-Wolf Z, Hall W Jr, Benacerraf B, Nadler L and Reiser H: Murine B7 antigen provides a sufficient costimulatory signal for antigen specific and MHC-restricted $\mathrm{T}$ cell activation. J Immunol 149: 3802-3808, 1992.

30. Andres PG, Howland, KC, Dresnek D, Edmondson S, Abbas AK and Krummel MF: CD28 signals in the immature immunological synapse. J Immunol 172: 5880-5886, 2004.

31. Purtic B, Pitcher LA, van Oers NS and Wülfing C: T cell receptor (TCR) clustering in the immunological synapse integrates TCR and costimulatory signaling in selected T cells. Proc Natl Acad Sci USA 102: 2904-2909, 2005. 